\title{
Explotação e exploração do conhecimento organizacional: inovações nos bares e restaurantes de Campina Grande
}

\begin{abstract}
No contexto dos processos organizacionais, visando à obtenção de vantagens competitivas, o conhecimento nas organizações pode ser analisado tanto na perspectiva de maior aproveitamento dos conhecimentos já existentes na organização (exploitation) ou com a ideia de prospecção, com o fim de se obter novos conhecimentos (exploration). Setores como o de bares e restaurantes sofrem um processo de concorrência muito elevada, exigindo um gerenciamento adequado do conhecimento, com perspectivas exploiter e/ou explorer, de modo que as inovações sejam rápidas, consistentes e adequadas ao mercado. Investigações que ilustrem as orientações exploiter e/ou explorer no ramo de bares e restaurantes não foram encontradas em bases de dados de pesquisas. Neste sentido, a presente investigação teve por objetivo analisar as orientações exploiter e/ou explorer, no tocante às inovações dos bares e restaurantes de Campina Grande. Para tal, a pesquisa aplicou um questionário referente à escala de orientação exploiter e/ou explorer (POPADIUK, 2010) em 50 estabelecimentos de Campina Grande. A pesquisa, de natureza descritiva, teve seus dados tratados de forma quantitativa. Estatísticas descritivas foram realizadas e analisadas, indicando não haver uma orientação prevalente de exploitation ou exploration, sinalizando, desse modo, a ambidestria na gestão do conhecimento das organizações investigadas.
\end{abstract}

Palavras-chave: Inovações; Bares e Restaurantes; Exploitation e Exploration.

\section{Exploitation and exploitation of organizational knowledge: innovations in the bars and restaurants of Campina Grande}

\begin{abstract}
In the context of organizational processes, in order to obtain competitive advantages, knowledge in organizations can be analyzed both in better use perspective of existing knowledge in the organization (exploitation) or the idea of exploration, with the intent to achieve new knowledge (exploration). Sectors such as bars and restaurants suffer a very high competitive process, requiring an adequate knowledge management, with exploiter prospects and/or explorer, so that innovations are fast, consistent and appropriate to the market. Researches that show the guidelines exploiter and/or explorer in the business of bars and restaurants were not found in research databases. In this sense, this research had an objective to analyze exploiter and/or explorer guidelines, with regard to innovations of bars and restaurants of Campina Grande. To this end, the research applied a questionnaire with the orientation scale exploiter and/or explorer (POPADIUK, 2010) in 50 establishments of Campina Grande. The research, descriptive, had their data processed quantitatively. Descriptive statistics were performed and analyzed, indicating there is no prevalent direction of exploitation or exploration, signalizing thereby the ambidexterity in knowledge management of the firms.
\end{abstract}

Keywords: Innovations; Bars and Restaurants; Exploitation and Exploration.

Topic: Empreendedorismo e Inovação

Reviewed anonymously in the process of blind peer.

Fábio de Oliveira Lucena

Universidade Presbiteriana Mackenzie, Brasil

http://lattes.cnpq.br/2014110572667710

f.lucena@ibest.com.br

Arthur Gomes de Barros Correia

Centro de Ensino Superior e Desenvolvimento, Brasil

http://lattes.cnpq.br/8600383424069298

arthurzinhobarros@gmail.com

Fabrícia Gomes Clemente

Faculdade de Ciências Sociais Aplicadas, Brasi

http://lattes.cnpq.br/1631086306587590

fgomesclemente@gmail.com

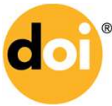

DOI: $10.6008 /$ SPC2179-684X.2016.001.0012
Received: 09/10/2015

Approved: 14/01/2016

Ricardo Kleber Braga Gonçalves Filho

Centro de Ensino Superior e Desenvolvimento, Brasil

http://lattes.cnpq.br/6278444721455083

ricardokleberb@gmail.com

Silvio Popadiuk

Faculdade de Economia Administração e Contabilidade da

Universidade São Paulo, Brasil

http://lattes.cnpq.br/1784002683973468

spopadiuk@gmail.com

Referencing this:

LUCENA, F. O.; CORREIRA, A. G. B.; CLEMENTE, F. G.; GONÇALVES FILHO, R. K. B.; POPADIUK, S.. Explotação e exploração do conhecimento organizacional: inovações nos bares e restaurantes de Campina Grande. Revista Brasileira de Administração Científica, v.7, n.1, p.176-189, 2016. DOI: http://doi.org/10.6008/SPC2179684X.2016.001.0012 


\section{INTRODUÇÃO}

As exigências cada vez mais crescentes por inovações com qualidade têm sido a tônica no mercado. Franklin (2010, p. 15) assevera que "como o grau de semelhança entre os produtos e serviços desenvolvidos é cada vez mais elevado, seja devido às inovações ou imitações", é importante que as empresas estejam alerta às dinâmicas competitivas nos ambientes onde estão insertas.

No contexto dos processos organizacionais, com vistas à obtenção de vantagens competitivas, o conhecimento nas organizações pode ser analisado sob abordagens distintas. De um lado, uma perspectiva de maior aproveitamento dos conhecimentos já existentes na organização, o que se denomina de explotação ou exploitation. Em outro sentido, com a ideia de prospecção, com o fim de se obter novos conhecimentos, capazes de fomentar inovações mais radicais, o conceito de exploração ou exploration (POPADIUK, 2007).

É crível, todavia, não haver uma dicotomia absoluta entre os conhecimentos já presentes na organização (explotação) e os novos conhecimentos obtidos externamente (exploração), sendo desejável o equilíbrio entre ambas fontes de conhecimento (MARCH, 1991; ZACK, 2002). Nesse sentido, as inovações que se refletem nos serviços e produtos requerem, quanto à gestão do conhecimento, políticas e ações que favoreçam os processos de explotação e exploração, com vistas a melhor criar e disseminar as inovações.

Setores como o de bares e restaurantes sofrem um processo de concorrência muito elevada, exigindo um gerenciamento adequado do conhecimento, com perspectivas exploiter e/ou explorer, de modo que as inovações sejam rápidas, consistentes e adequadas ao mercado.

Tendo por premissa a necessidade de gerir o conhecimento organizacional que favoreça a disseminação de inovações nas organizações e considerando o acirramento competitivo de setores como o de bares e restaurantes, a presente investigação se debruçou sobre a seguinte questão: Como os processos de explotação e exploração influenciam as inovações, em produtos e serviços, no segmento de bares e restaurantes de Campina Grande?

Para responder a essa questão, adotou-se como objetivo geral da presente pesquisa analisar as orientações exploiter e/ou explorer, no tocante às inovações dos bares e restaurantes de Campina Grande. Para atender este objetivo, foram visados três objetivos específicos, quais sejam: (a) descrever os serviços e produtos de cada estabelecimento (bar ou restaurante); (b) analisar as inovações de serviços e produtos dos bares e restaurantes campinenses, procurando estabelecer as relações com os processos de explotação e exploração.

A investigação procurou um modelo que informasse as relações existentes entre os processos de explotação e exploração e as inovações de serviços e produtos do ramo de bares e restaurantes, o que pode subsidiar as decisões estratégicas dos gestores no tocante à gestão do conhecimento.

Devido à complexidade do tema e além de se tratar de um assunto relativamente recente, não se encontra uma pesquisa específica sobre a orientação exploiter e/ou explorer no ramo econômico investigado, o que propicia uma certa contribuição acadêmica à área de pesquisa. 


\section{REVISÃO TEÓRICA}

No contexto dinâmico das organizações o conhecimento pode ser entendido como um sistema vivo, que cresce e se altera por meio da interação com o meio ambiente (DAVENPORT; PRUSAK, 2004).

Por conhecimento, pode-se entender como uma combinação fluída de experiência condensada, que inclui valores, informação contextual e insight derivado de experiência, a que permite uma estrutura para a avaliação e incorporação de novas experiências e informações (DAVENPORT; PRUSAK, 2004). Nas organizações, o conhecimento está presente não apenas em documentos, como também em rotinas, processos, práticas e normas organizacionais.

Entre vários dos aspectos que devem ser observados pelas organizações a fim de que as mesmas desenvolvam adequadamente seus conteúdos estratégicos, encontra-se o gerenciamento dos ativos de conhecimento. A esse respeito, Malhotra, ao contribuir para a fundamentação de um relatório da Unidas (2003), declara que os ativos do conhecimento se constituem como a fonte de competências das organizações, sendo, portanto, vitais para a sobrevivência e competitividades das mesmas. Explica ainda que as questões relativas à eficiência e à eficácia dos ativos do conhecimento são relacionadas ao retorno dos investimentos, ou seja, a maneira como a organização emprega esses ativos (explotação e exploração) contribui para a sua vantagem competitiva. Os ativos de conhecimento se localizam entre os recursos intangíveis das organizações (HITT; IRELAND; HOSKISSON, 2008). Um quadro elucidativo de tais recursos pode ser observado em Popadiuk (2007), como ilustra a Figura 1.

A gestão do conhecimento auxilia nos processos de conhecimento e na sua criação, tendo uma relação mais estreita "com a gestão de informações, tecnologias e inovações, assim como com a gestão de competências dentro das organizações com ênfase na absorção de conhecimento tácito" (MADEIRA et al., $2013, s / p)$.

O gerenciamento do conhecimento é importante para preservar o patrimônio valioso, aprender novas ideias, resolver problemas, criar competências centrais, entre outras questões importantes para empresas inovadoras (LIAO, 2003).

É preciso considerar, na gestão do conhecimento, que os recursos intangíveis não são simples de serem potencializados numa organização, devido à subjetividade associada aos seus conteúdos. Por exemplo, quais são efetivamente os conhecimentos tácitos dos colaboradores? Como tais conhecimentos, combinados com os conhecimentos explícitos, contribuem para as inovações nos produtos e serviços da organização?

A partir dos pressupostos da aprendizagem organizacional, March (1991) propõe uma abordagem no qual são desenvolvidos dois conceitos básicos: explotação e exploração. Para o autor, explotação inclui aspectos como refinamento, escolha, produção, eficiência, seleção, implementação e execução, representando, desse modo, a extensão das competências já existentes, o que implica retornos previsíveis e mais rápidos. 
Prosseguindo, March (1991) afirma que exploração é busca de novas alternativas, associado a termos como pesquisa, risco, experimentação, jogo, flexibilidade, descoberta, inovação, conduzindo, destarte, a retornos incertos, imprevisíveis, de longo prazo, e frequentemente negativos. March apresenta dois modelos: o primeiro explicita como se dá a aprendizagem mútua entre os indivíduos e a organização (por meio do código organizacional); o segundo modelo relaciona aprendizagem e vantagem competitiva. Argumenta finalmente que processos adaptativos, por explotação, tendem a se tornar mais eficazes em curto prazo, com retornos previsíveis, porém apresentam comprometimento competitivo em longo prazo.

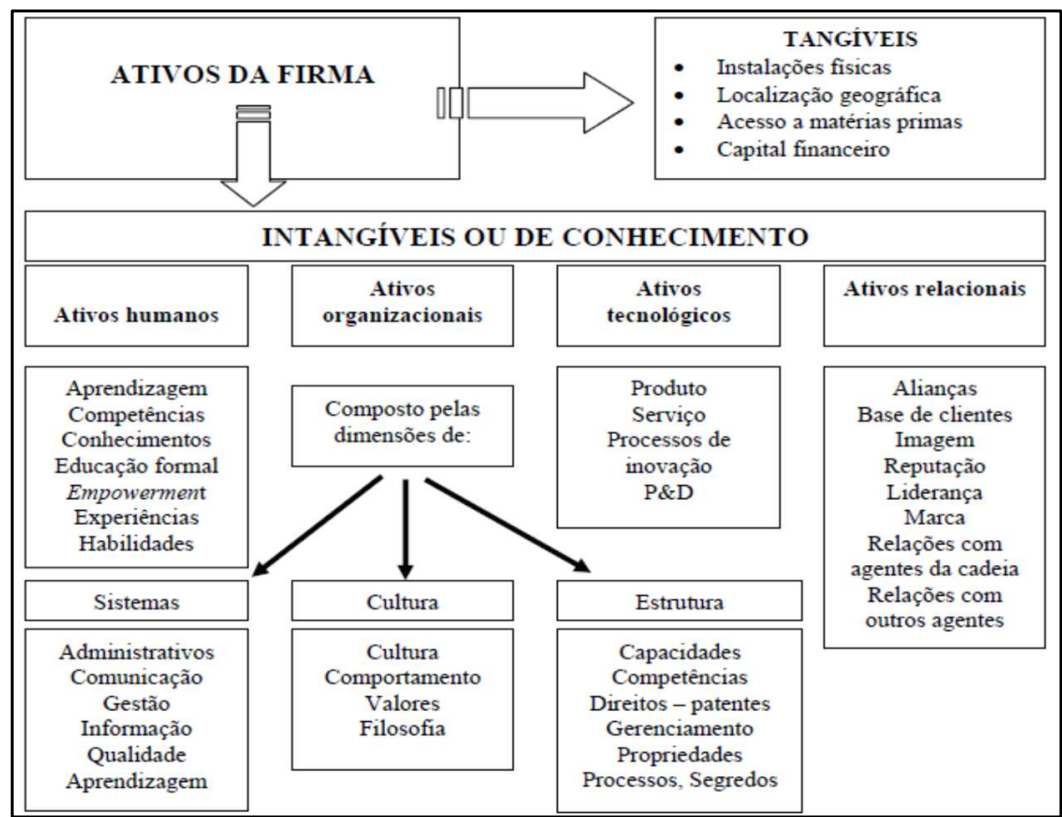

Figura 1: Categorização de ativos tangíveis e intangíveis. Fonte: Popadiuk (2007).

Finalmente, conclui que é necessário estabelecer um equilíbrio entre explotação e exploração, com vistas a promover a sobrevivência das organizações. Os processos adaptáveis, através do explotação, em face do emprego mais célere dos conhecimentos, no lugar da exploração, apresentam uma tendência de ser mais efetivos no curto prazo (MARCH, 1991), porém pode levar as empresas a se atrelarem a soluções sub-ótimas e, desse modo, deixá-las despreparadas a ambientes com mudanças mais intensas (FANG; LEE; SCHILLING, 2010).

Popadiuk e Vidal (2009) afirmam que há certos atributos dos ambientes interno e externo da organização associados à explotação e exploração, quais sejam: (a) ambiente interno: conhecimento organizacional por meio de geração de ideias, conhecimento tácito e seu compartilhamento; aprendizagem interna; eficiência organizacional, que pode ser melhorada através de refinamento de produtos ou nas rotinas de execução; orientação estratégica através de visão de curto e longo prazo sobre o negócio; custos de pesquisa e desenvolvimento; inovações tanto em produtos e processos, entre outros atributos; (b) ambiente externo: concorrência, caracterizada pela de entrada de novos concorrentes e ou pela ação dos concorrentes com características e estruturas semelhantes; parcerias através de criação de alianças, interação com parceiros, aumento de relacionamento externo e grau de dependência dos sócios. 
A dicotomia entre os dois processos de aprendizagem organizacional em questão não é recomendável, pois a manutenção do apropriado equilíbrio entre exploração e explotação é um fator primordial para a sobrevivência e prosperidade das organizações (MARCH, 1991; ZACK, 2002).

Todavia, o processo de equilíbrio entre exploração e explotação é ambíguo (MOM; BOSCH; VOLBERDA, 2003) e complicado, em face do próprio desequilíbrio da aprendizagem (LEVINTHAL; MARCH, 1993). A dificuldade se torna mais evidente pelo fato de ambas as orientações requererem os mesmos recursos, que são escassos (NISHIMURA, 2012).

Os estudos, aprendizados, aperfeiçoamentos e aquisição de novos conhecimentos são fundamentais tanto para explotação como exploração. Ao mesmo tempo, as diferenças entre os dois conceitos dizem respeito se o novo aprendizado ocorre ao longo da mesma trajetória do antigo aprendizado ou mediante uma trajetória completamente diferente (GUPTA; SMITH; SHALLEY, 2006).

Numa tentativa de melhor tradução para português, sem perda semântica significativa e contextualizando no ambiente empresarial, Popadiuk (2007) substitui a palavras explotação por aproveitamento e exploração por prospecção. Entretanto, não há um consenso sobre uma definição dos termos, pois, alguns autores empregam termos com diferentes nomenclaturas, com significados semelhantes. Como exemplo, Nelson e Winter (1982) empregaram os termos refinamento e invenção, que estão associados a explotação e exploração, respectivamente. Popadiuk (2007), por sua vez, sintetiza as características das duas abordagens de utilização do conhecimento (Quadro 1).

Quadro 1: Síntese de características associadas com exploration e exploitation.

\begin{tabular}{|c|c|c|c|}
\hline \multicolumn{2}{|c|}{$\begin{array}{l}\text { EXPLOITATION (APROVEITAMENTO) DE } \\
\text { CONHECIMENTOS }\end{array}$} & \multicolumn{2}{|c|}{ EXPLORATION (PROSPECÇÃO) DE CONHECIMENTOS } \\
\hline Refinamento & Orientação para produto & Pesquisa & Estrutura mais flexível \\
\hline Escolha & Conhecimento codificado & Busca & Redes com tamanho \\
\hline Foco em produção & Alianças formais & Mais riscos & limitado \\
\hline Foco em eficiência & Mais contratos & Experimentação & Novas rotinas \\
\hline Seleção & Busca de reputação & Variedade de ideias & Novos aprendizados \\
\hline Implementação & Mais confiança & Novos conhecimentos & Longo prazo \\
\hline Execução & Redes densas & Conhecimento tácito & Capacidades dinâmicas \\
\hline Design dominante & Formalização & Parcerias & Diferenciação \\
\hline Redução de custos & Estabilização & Novas capacitações & Heterogeneidade \\
\hline Controles maiores & Competição maior & Custos maiores & Amplitude \\
\hline Rotinas, regras & Curto prazo & Inovação radical & Mais incerteza \\
\hline Ganhos de escalas & Path dependence & Orientação $p /$ tecnologia & Visão de futuro \\
\hline Inovação incremental & Pouca variação & Novos entrantes & Descontinuidade \\
\hline Subutilização de recursos & Padronizações & Alianças fracas Uso limitado de & Ruptura \\
\hline Homogeneidade & Continuidade & contratos & Jogo \\
\hline Profundidade & Obsolescência & Interações mais próximas & Doble-loop \\
\hline Menores riscos & Laços duráveis & Maior confiança & \\
\hline Conservadorismo & Recompensas & Retornos incertos & \\
\hline Single-loop & Sociedades profissionais & & \\
\hline
\end{tabular}

Fonte: Popadiuk (2007)

As diversas ideias apresentadas nesta seção sinalizam no sentido de que o adequado gerenciamento do conhecimento é um desafio para as organizações, pois o direcionamento enfático para uma das abordagens traz determinadas consequências negativas: se a ênfase é em explotação, a obsolescência organizacional pode ocorrer; do contrário, a ênfase em exploração pode gerar muitos retornos incertos. 0 equilíbrio, como advogam March (1991) e Zack (2002), e desejável em virtude das situações ora colocadas. 
Consigne-se que quando a organização é capaz de simultaneamente aproveitar as competências e explorar novas oportunidades, indicando uma certa ambivalência do uso do conhecimento, tem-se uma ambidestria (RAISCH et al., 2009). Na ambidestria, a organização apresenta a capacidade de desenvolver e pesquisar novos produtos e/ou serviços concomitantemente com o aperfeiçoamento dos já existentes (SILVEIRA-MARTINS; ROSSETTO, 2014).

Cabe registrar que tanto a aprendizagem organizacional como as inovações ocorrem de forma simultânea, porém com características próprias. Mesmo que os dois fenômenos se processem de forma praticamente conjunta, "é proveitoso considerar a inovação e a aprendizagem organizacional como dois fenômenos distintos e interpretá-los de dentro de duas teorias diferentes" (SUNDBO, 1997, p. 437). A inovação representa a mudança no negócio em face da adição de um novo elemento ou uma nova combinação de velhos elementos, segundo a abordagem schumpeteriana (SUNDBO; GALLOUJ, 1998).

Em relação aos impactos gerados, a inovação pode ser radical ou incremental. Uma inovação é tida como radical quando há um desenvolvimento ou introdução de um novo produto, processo ou forma de organização da produção inteiramente nova (LEMOS, 2000), o que causa uma ruptura significativa no negócio. Tal tipo de inovação está normalmente associada ao processo de exploração do conhecimento (MARCH, 1991).

Por outro lado, a inovação incremental refere-se à introdução de qualquer tipo de melhoria em um produto, processo ou organização produtiva, dentro de uma empresa, sem alteração significativa da estrutura industrial (do segmento econômico) (FREEMAN, 1998). Segundo Abernathy e Clark (1985), as empresas tendem a inovar de forma incremental, a partir das suas competências já estabelecidas, correspondendo ao processo de explotação de March (1991).

Com uma perspectiva mais ampla do que a visão estritamente econômica, Reichert, Camboim e Zawislak (2015, p. 165) afirmam que a inovação é resultado da capacidade das empresas, tendo por referência "os padrões tecnológicos e de mercado em cada ramo de atividade, de absorver, adaptar e transformar conhecimento em tecnologia e esta em rotinas operacionais, gerenciais e comerciais que levem as empresas a atingir um desemepenho superior." Os autores acrescentam que a inovação deve ser condiderada a partir de todas as capacidades internas das firmas e não somente a partir de indicadores tecnológicas.

De acordo com Sundbo e Gallouj (1998), há quantro tipos de inovações: inovações de produto; inovações de processo; inovações organizacionais ou gerenciais e inovações de mercado. Em outra abordagem, que contempla a capacidade de inovação da organização, as inovações se dão nos níveis tecnológico, operacional, gerencial e comercial (Zawislak et al., 2013).

Hauknes (1998) acrescenta uma nova compreensão de inovação em serviços, quando afirma que esta pode ser entendida como um processo de compreensão de capacidades obtidas nas relações específicas com o consumidor do serviço. Neste sentido, o cliente do serviço passa a também ser um elemento central da análise das inovações em serviços. 


\section{METODOLOGIA}

O presente estudo compreendeu procedimentos que visaram à análise das orientações exploiter e/ou explorer, no tocante às inovações dos bares e restaurantes de Campina Grande. Como enfatizou uma descrição analítica de tais aspectos, elucidando as características do fenômeno estudo, em uma perspectiva organizacional e com uma orientação para um segmento específico, este tipo de estudo, segundo Sekaran (2003), é considerado um estudo descritivo.

A investigação é considerada também como uma pesquisa de campo, pois buscou obter dados diretamente nos estabelecimentos investigados. Inicialmente foi feita uma revisão bibliográfica acerca do tema em pauta, procurando estabelecer proposições acerca das relações entre as práticas de explotação e exploração e inovações em produtos e serviços.

A partir dos pressupostos teóricos e de uma escala de mensuração para orientação explotaçãoexploração de conhecimento das empresas, desenvolvida por Popadiuk (2010), foi aplicado um questionário (Apêndice A) nas organizações investigadas, numa escala tipo Likert com sete pontos. A escala contém seis atributos, como ilustra a Tabela 1.

Tabela 1: Nomenclaturas com significados semelhantes a explotação e exploration.

\begin{tabular}{c|c}
\hline ATRIBUTO & QUANTIDADE DE ITENS \\
\hline Conhecimento organizacional & 10 \\
\hline Eficiência organizacional & 8 \\
\hline Orientação estratégica & 2 \\
\hline Inovação & $8^{\mathrm{b}}$ \\
\hline Competição & 8 \\
\hline Parcerias & 8 \\
\hline TOTAL & 44 \\
\hline
\end{tabular}

Fonte: Adaptado de Popadiuk (2010). Notas: (a) Algumas alterações foram realizadas, pois o perfil do instrumento era mais dirigido a indústrias; (b) b) Foi removido o item "desenvolvimento de protótipos". Logo, na escala original tinha 9 itens, que agora passou a contar com 8.

Antes da aplicação do questionário, foi desenvolvido um estudo piloto em onze organizações, como vistas a avaliar a validade de face do instrumento de pesquisa. (HAIR JR. et al., 2009). Com as devidas correções, foi aplicado um questionário a 50 (cinquenta) estabelecimentos. Os dados foram tratados de modo quantitativo na planilha SPSS, aplicando-se análise fatorial exploratória (HAIR JR. et al., 2010).

A abordagem das organizações foi através de contato pessoal com os gestores, de modo que fosse possível alcançar a quantidade de 50 respondentes. A amostra, portanto, foi por acessibilidade e, por conseguinte, não probabilística. Não há cadastros disponíveis sobre o tamanho da população (bares e restaurantes) em Campina Grande, porém estima-se uma quantidade superior a 200 unidades formalmente constituídas. Registre-se que, antes da pesquisa de campo em si, o presente projeto de pesquisa, com os requisitos que a Res. 466/2012 exige, foi submetido ao Comitê de Ética na Pesquisa do CESED para fins de aprovação.

\section{RESULTADOS DA PESQUISA}

\section{Estatística descritiva}


Inicialmente, são apresentados os resultados das respostas e um breve tratamento estatístico descritivo. Os resultados que se seguem dizem respeito aos questionários aplicados aos 50 respondentes. Algumas informações preliminares acerca dos respondentes foram colhidas, ilustradas na Tabela 2. A maior parte dos respondentes, $56 \%$, é formada pelos próprios proprietários dos bares e restaurantes. Verificou-se também que $62 \%$ dos participantes eram do sexo masculino.

Quanto à formação acadêmica, uma parcela pequena, 12 por cento, tinham pós-graduação, enquanto que $46 \%$ declararam possuir curso superior e $42 \%$, ensino médio. Ressalte-se que, apesar da formação acadêmica contribuir para um melhor desempenho dos negócios, isto pode não ter relação com o sucesso em empreendimentos.

Tabela 2: Informações preliminares sobre os respondentes.

\begin{tabular}{|c|c|c|c|c|c|c|}
\hline \multirow{3}{*}{ Cargo na empresa } & \multicolumn{3}{|c|}{ Proprietário } & \multicolumn{3}{|c|}{ Gerente } \\
\hline & \multicolumn{2}{|c|}{\begin{tabular}{l|l} 
Quantidade &
\end{tabular}} & Frequência relativa & \multicolumn{2}{|c|}{ Quantidade } & Frequência relativa \\
\hline & \multicolumn{2}{|l|}{28} & $56 \%$ & \multicolumn{2}{|c|}{22} & $44 \%$ \\
\hline \multirow{3}{*}{ Gênero } & \multicolumn{3}{|c|}{ Masculino } & \multicolumn{3}{|c|}{ Feminino } \\
\hline & \multicolumn{2}{|l|}{ Quantidade } & Frequência relativa & \multicolumn{2}{|c|}{ Quantidade } & Frequência relativa \\
\hline & \multirow{2}{*}{\multicolumn{2}{|c|}{$\frac{31}{\text { Pós-graduacão }}$}} & $62 \%$ & \multicolumn{2}{|c|}{19} & $38 \%$ \\
\hline \multirow[t]{3}{*}{ Formação acadêmica } & & & \multicolumn{2}{|c|}{ Superior } & \multicolumn{2}{|c|}{ Ensino médio } \\
\hline & Quant. & F. rel. & Quant. & F. rel. & Quant. & F. rel. \\
\hline & 6 & $12 \%$ & 23 & $46 \%$ & 21 & $42 \%$ \\
\hline
\end{tabular}

Foram obtidos também dados relativos aos estabelecimentos, incluindo quantidade de funcionários, número estimado de clientes/dia, posição estratégica vista pelo respondente no mercado e tipo de organização, como apresenta a Tabela 3. Observa-se que a maioria dos estabelecimentos visitados, 56\%, têm entre 10 e 49 colaboradores. Apenas quatro unidades visitadas informaram haver mais de 50 colaboradores. Quanto ao número estimado de clientes que frequentam os estabelecimentos por dia, $86 \%$ dos respondentes declararam que há uma frequência superior a 50 clientes/dia.

Tabela 3: Informações preliminares sobre os estabelecimentos.

\begin{tabular}{|c|c|c|c|c|c|c|c|c|}
\hline \multirow{3}{*}{$\begin{array}{l}\text { Quantidade de funcionários } \\
\text { (fixos, temporários, terceirizados) }\end{array}$} & \multicolumn{2}{|c|}{ Até nove } & \multicolumn{3}{|c|}{ De 10 a 49} & \multicolumn{3}{|c|}{ De 50 acima } \\
\hline & Quant. & \multicolumn{2}{|c|}{ F. rel. } & Quant. & F. rel. & \multicolumn{2}{|c|}{ Quant. } & F. rel. \\
\hline & 18 & \multirow{2}{*}{\begin{tabular}{l|}
\multicolumn{2}{c}{$36 \%$} \\
49
\end{tabular}} & & 28 & $56 \%$ & \multicolumn{2}{|r|}{4} & $8 \%$ \\
\hline \multirow{3}{*}{ № estimado de clientes que frequentam por dia } & Até 49 & & \multicolumn{2}{|c|}{ De 50 a 199} & \multicolumn{2}{|c|}{ De 200 a 499} & \multicolumn{2}{|c|}{ De 500 acima } \\
\hline & Quant. & F. rel. & Quant. & F. rel. & Quant. & F. rel. & Quant. & F. rel. \\
\hline & 7 & $14 \%$ & 27 & $54 \%$ & 15 & $30 \%$ & 1 & $2 \%$ \\
\hline \multirow{3}{*}{ Posição estratégica no mercado } & \multicolumn{2}{|c|}{ Líder } & \multicolumn{2}{|c|}{ 2a posição } & \multicolumn{2}{|c|}{ 3a posição } & \multicolumn{2}{|c|}{ Não sabe } \\
\hline & Quant. & F. rel. & Quant. & F. rel. & Quant. & F. rel. & Quant. & F. rel. \\
\hline & 18 & $36 \%$ & 12 & $24 \%$ & 3 & $6 \%$ & 17 & $34 \%$ \\
\hline \multirow{3}{*}{ Tipo de organização } & \multicolumn{2}{|c|}{ Familiar } & \multicolumn{2}{|c|}{ Franquia } & \multicolumn{2}{|c|}{ Filial } & \multicolumn{2}{|c|}{ Matriz } \\
\hline & Quant. & F. rel. & Quant. & F. rel. & Quant. & F. rel. & Quant. & F. rel. \\
\hline & 28 & $56 \%$ & 11 & $22 \%$ & 5 & $10 \%$ & 6 & $12 \%$ \\
\hline
\end{tabular}

No que se refere à percepção da posição estratégica no mercado, a Tabela 3 informa que 36\% dos participantes acreditam serem líderes; 24\%, 2a posição no mercado; seis por cento, 3a posição; e 34\%, não sabem. Essa questão mereceria uma outra pesquisa no segmento para avaliar as questões de estratégia no mercado. 
Quanto ao tipo de organização, verificou-se que $56 \%$ dos estabelecimentos são de natureza familiar. Ainda a respeito das inovações recentes que as empresas implementaram entre 2013 e 2014, os participantes responderam alguns tipos de inovações, conforme a Tabela 4.

Tabela 4: Inovações recentes (2013-2014) implementadas nos estabelecimentos.

\begin{tabular}{l|c}
\hline \multicolumn{1}{c|}{ Área da inovação } & Quantidade \\
\hline Pratos (alimentos) e/ou bebidas & 42 \\
\hline Serviços (formas novas de servir os alimentos e bebidas) & 18 \\
\hline Novas formas de entregas & 12 \\
\hline Entretenimentos (música, shows, playgrounds infantis etc.) & 13 \\
\hline Ambiente físico (layout, mesas etc.) & 15 \\
\hline Outras & 5 \\
\hline
\end{tabular}

De acordo com a Tabela 4, constata-se que a maior parte das inovações são relativas a pratos (alimentos) e/ou bebidas, o que, em certo sentido, era esperado pela natureza do negócio investigado. Houve a incidência também de inovações em se tratando de serviços, tanto na forma de servir os alimentos e bebidas, quanto na maneira de se entregar os produtos e serviços. Essas inovações são consideradas incrementais, como elucidam Freeman (1998) e Abernathy e Clark (1985) e estão situadas predominantemente nos níveis de capacidade de operação e comercial, na visão de Zawislak et al. (2013).

No que se refere ao objeto do estudo, ou seja, sobre a orientação exploiter e explorer de conhecimentos, calculou-se a média, a mediana e o desvio-padrão de todos os itens da planilha. As Tabelas $5,6,7,8,9$ e 10 relacionam estes valores por dimensão estudada.

Tabela 5: Práticas de conhecimento - média, mediana e desvio-padrão.

\begin{tabular}{ccccccccccc}
\hline DIMENSÃO & PC_1 & PC_2 & PC_3 & PC_4 & PC_5 & PC_6 & PC_7 & PC_8 & PC_9 & PC_10 \\
\hline MÉDIA & 5,02 & 4,28 & 4,66 & 5,86 & 5,36 & 5,66 & 5,4 & 5,48 & 5,04 & 5,6 \\
\hline MEDIANA & 5 & 5 & 5 & 6 & 6 & 6 & 5,5 & 6 & 5 & 6 \\
\hline DESVIO-PADRÃO & 1,55 & 1,98 & 1,80 & 1,31 & 1,70 & 1,21 & 1,32 & 1,33 & 1,50 & 1,34 \\
\hline
\end{tabular}

Em relação à Tabela 5, observa-se que a média que apresentou a menor média foi a PC_2 (uso de novas fontes de conhecimentos de parceiros), com um valor de 4,28 . Nota-se que a mediana mais frequente foi 6. Quanto ao desvio-padrão, o valor variou de 1,21 a 1,98, indicado haver uma variação significativa na escala de 1 a 7. A média mais alta neste grupo de variáveis foi de 5,86, relativa à PC_4 (utilização do conhecimento já existente na empresa), indicando, desse modo, uma prevalência da orientação exploiter (MARCH, 1991; POPADIUK, 2010).

Tabela 6: Práticas de inovação - média, mediana e desvio-padrão.

\begin{tabular}{cccccccccc}
\hline DIMENSÃO & PI_1 & PI_2 & PI_3 & PI_4 & PI_5 & PI_6 & PI_7 & PI_8 & PI_9 \\
\hline MÉDIA & 5,1 & 4,68 & 4,64 & 4,36 & 4,3 & 4,12 & 5,02 & 5,28 & 3,76 \\
\hline MEDIANA & 6 & 5 & 5 & 4 & 5 & 4 & 5,5 & 5 & 3,5 \\
\hline DESVIO-PADRÃO & 1,63 & 1,74 & 1,92 & 2,09 & 2,00 & 1,99 & 1,92 & 1,57 & 2,11 \\
\hline
\end{tabular}

Ao se apreciar a Tabela 6 ou o Gráfico 1, constatou-se que a média mais baixa foi de 4,12, relacionada à PI_6 (foco de inovações radicais em tecnologias). A mediana da variável PI_1 (foco em produtos ou processos totalmente novos) foi muito alta, ou seja, 6, numa escala de 1 a 7. Nesse sentido, a adoção de inovações com produtos ou processos novos indica haver uma orientação explorer de conhecimento, 
consoante March (1991). Verificou-se que a variável PI_9 (participação agressiva de alianças tecnológicas) apresentou a menor média entre as variáveis relativas a práticas de inovação, embora tenha apresentado o maior desvio-padrão $(2,11)$ entre os demais. Isto, em certo sentido, contradiz a orientação explorer.

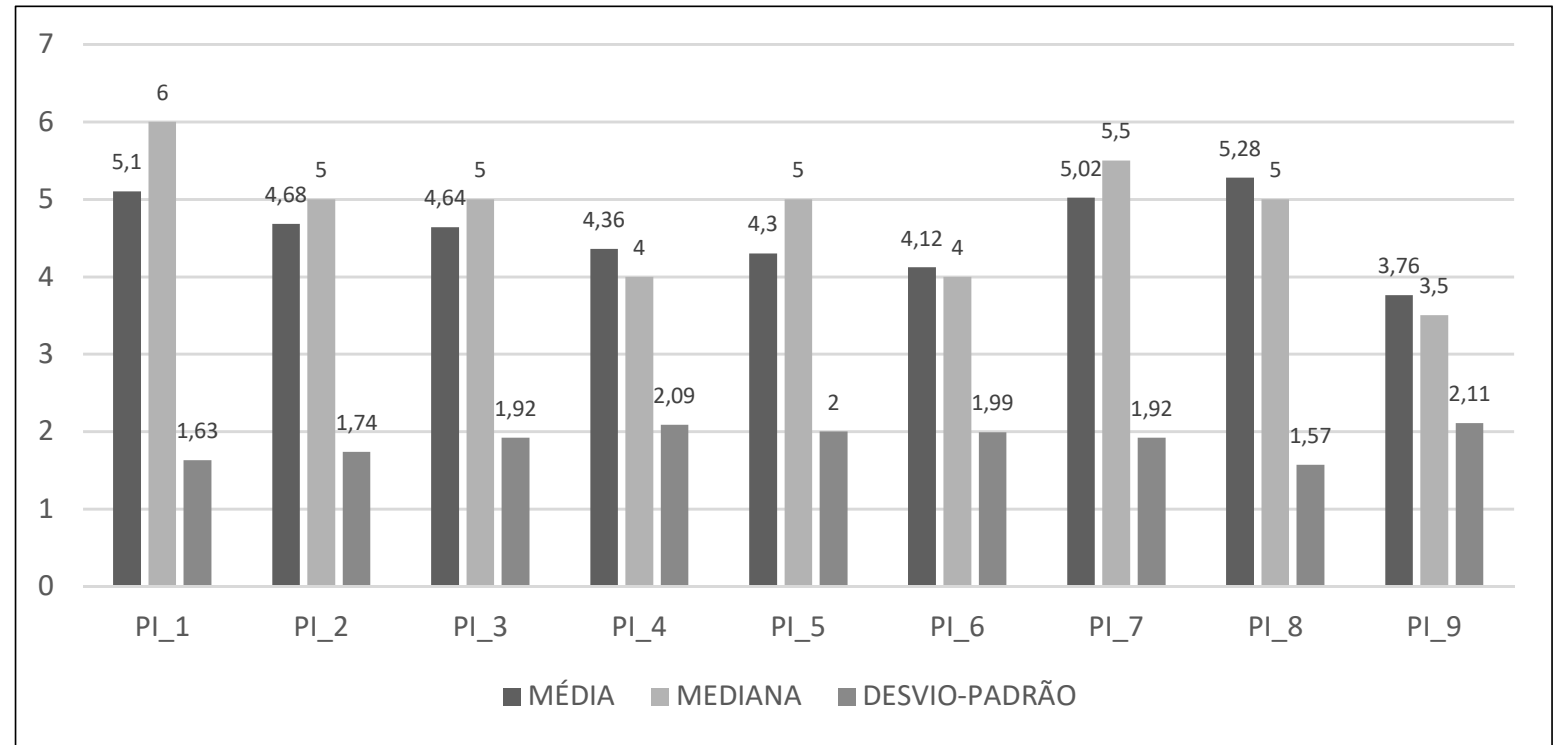

Gráfico 1: Práticas de inovação - média, mediana e desvio-padrão.

Tabela 7: Competição - média, mediana e desvio-padrão.

\begin{tabular}{ccccccccc}
\hline DIMENSÃO & CP_1 & CP_2 & CP_3 & CP_4 & CP_5 & CP_6 & CP_7 & CP_8 \\
\hline MÉDIA & 4,24 & 4 & 4,86 & 4,64 & 4,12 & 4,16 & 2,92 & 3,18 \\
\hline MEDIANA & 5 & 4 & 5 & 5 & 4,5 & 4 & 3 & 3 \\
\hline DESVIO-PADRÃO & 2,11 & 1,87 & 1,91 & 2,01 & 2,13 & 1,99 & 1,84 & 1,95 \\
\hline
\end{tabular}

No que tange aos dados da Tabela 7, verificou-se, por exemplo, que a média da variável CP_2 (existência de produtos ou processos substitutos) foi de 4 , com uma mediana de 4 também. A menor média verificada foi de 3,18, relativa à variável CP_8 (competição por preço é o ponto alto de nossa empresa). Este aspecto indica que o preço não representa o critério competitivo da maioria das organizações investigadas, indicando haver um foco em outras prioridades competitivas, como Corrêa e Corrêa (2012) em face do tradeoff nas decisões estratégicas.

Observando a Tabela 8 e o Gráfico 2, constatou-se que a média da variável OE_1 (visão estratégica focada no presente) foi 5,42. Tal aspecto reforça a orientação exploiter, pois as decisões estratégicas que visam a dar respostas ao mercado no curto prazo revelam um maior aproveitamento dos recursos estratégicos, no lugar de explorar outros recursos (BARNEY; CLARK, 2007). O desvios-padrão da OE_1 e da OE_2 (estratégias relacionadas com o curto prazo) foram muito próximos, ou seja, 1,77 e 1,71, respectivamente.

Tabela 8: Orientação estratégica - média, mediana e desvio-padrão.

\begin{tabular}{ccc}
\hline DIMENSÃO & OE_1 & OE_2 \\
\hline MÉDIA & 5,42 & 4,84 \\
\hline MEDIANA & 6 & 5 \\
\hline DESVIO-PADRÃO & 1,77 & 1,71 \\
\hline
\end{tabular}




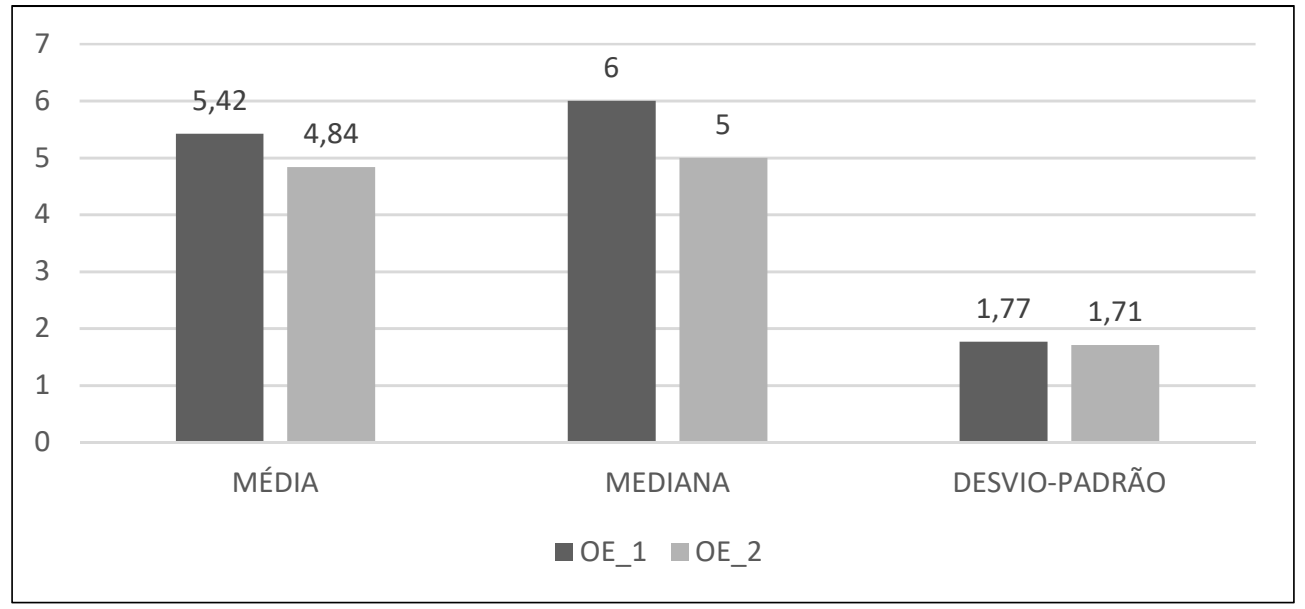

Gráfico 2: Orientação estratégica - média, mediana e desvio-padrão.

Tabela 9: Eficiência - média, mediana e desvio-padrão.

\begin{tabular}{cccccccc}
\hline DIMENSÃO & EF_1 & EF_2 & EF_3 & EF_4 & EF_5 & EF_6 & EF_7 \\
\hline MÉDIA & 5,46 & 6,38 & 6,08 & 5,04 & 5,42 & 6,06 & 5,88 \\
\hline MEDIANA & 6 & 7 & 6 & 5 & 6 & 6,5 & 6 \\
\hline DESVIO-PADRÃO & 1,53 & 0,83 & 1,08 & 1,87 & 1,65 & 1,28 & 1,44 \\
\hline
\end{tabular}

No que atine aos dados da Tabela 9, nota-se que houve um valor médio muito alto da variável EF_2 (grau de dependência dos parceiros externos), com mediana no valor de 6 (escala de 1 a 7). Percebe-se que a dependência dos fornecedores se configura como um aspecto relevante no segmento estudado.

Tabela 10: Parcerias - média, mediana e desvio-padrão.

\begin{tabular}{ccccccccc}
\hline DIMENSÃO & PA_1 & PA_2 & PA_3 & PA_4 & PA_5 & PA_6 & PA_7 & PA_8 \\
\hline MÉDIA & 4,9 & 3,54 & 3,3 & 5,48 & 4,64 & 4,22 & 4,44 & 4,32 \\
\hline MEDIANA & 5 & 4 & 3 & 6,5 & 5 & 5 & 5 & 5 \\
\hline DESVIO-PADRÃO & 2,10 & 1,89 & 2,04 & 1,93 & 2,13 & 2,15 & 2,16 & 1,94 \\
\hline
\end{tabular}

Os dados da Tabela 10, indicam, por exemplo, um valor médio baixo da variável PA_3 (uso de contratos nas relações com parceiros externos), com 3,3, embora tenha apresentado um desvio-padrão muito alto $(2,04)$. Esta questão revela que há uma tendência para não haver a adoção de contratos de longo prazo (parcerias) com os fornecedores.

\section{Análise fatorial exploratória: tentativa e limitação}

Mesmo com o número reduzido da amostra, tentou-se aplicar uma análise fatorial exploratório. Utilizando a ferramenta SPSS e tendo por base os dados referentes a variáveis que definem a orientação explotação-exploração de conhecimento das empresas (POPADIUK, 2010), foi rodada a planilha inicialmente com todos os indicadores, obtendo-se os resultados da Tabela 11.

Tabela 11: KMO and Bartlett's Test.

\begin{tabular}{lrr}
\hline Kaiser-Meyer-Olkin Measure of Sampling Adequacy. &, 291 \\
\hline \multirow{2}{*}{ Bartlett's Test of Sphericity } & Approx. Chi-Square & 1889,470 \\
\cline { 2 - 3 } & df & 946 \\
\cline { 2 - 3 } & Sig. &, 000 \\
\hline
\end{tabular}


O Kaiser-Meyer-Olkin (KMO), ou medida de adequação da amostra, indica o grau de ajuste dos dados à análise fatorial exploratória. Valores abaixo de 0,5 indicam a inadequação do método da análise fatorial exploratória ao conjunto de dados. Também informam que a soma das correlações parciais dos itens avaliados é bastante alta em relação ao somatório das correlações totais (PASQUALI, 1999). Como o resultado foi de 0,291 , verificou-se, portanto, que os dados levantados nesta pesquisa não se adequam à AFE. Desse modo, a análise fatorial exploratória restou prejudicada.

Algumas hipóteses podem ser colocadas em face dessa incompatibilidade: (a) o número das observações (neste caso, 50) foi pequeno; (b) alguns itens não estão adequadamente situados no modelo proposto e, desse modo, teriam que ser removidos; (c) outros itens deveriam ser incorporados aos ao modelo.

\section{CONSIDERAÇÕES FINAIS}

A presente investigação teve por objetivo primário analisar as orientações exploiter e/ou explorer, no tocante às inovações dos bares e restaurantes de Campina Grande. Nesse sentido, aplicou-se um questionário que revelou alguns aspectos, a saber: (a) uma parte considerável dos respondentes (36\%) acredita que seus estabelecimentos são líderes, no que atine à posição estratégica no mercado. Por outro lado, 34\% não sabem. Essa questão indica a necessidade de uma outra pesquisa no segmento para avaliar as questões de estratégia no mercado; (b) quanto ao tipo de organização, verificou-se que $56 \%$ dos estabelecimentos são de natureza familiar; (c) quanto às principais inovações reveladas pelos respondentes, verificou-se a prevalência daquelas relacionadas a pratos (alimentos) e/ou bebidas - aspecto que era previsível, por se tratar do tipo de negócio investigado. Todavia, foram informadas também inovações em serviços (novas formas de servir os alimentos e bebidas), como novas formas de entregas e entretenimentos. Estes foram as principais inovações indicadas. 0 total de inovações foi de 105, uma média de 2,1 inovações por estabelecimento. Isto revela uma postura de inovação no segmento.

Tentou-se aplicar a análise fatorial exploratória, mas esta se revelou prejudicada, porque o teste de Kaiser-Meyer-Olkin (KMO), também chamado de medida de adequação da amostra ficou abaixo de 0,5. Desse modo o método da AFE não se adequa ao conjunto dos dados colhidos. Isto impediu, por exemplo, de definir fatores que pudessem agrupar as variáveis analisadas ou mesmo confirmar a disposição atual. Algumas suposições podem ser colocadas em face dessa incompatibilidade, a saber: (a) o número das observações (neste caso, 50) foi reduzido; (b) alguns itens não estão adequadamente situados no modelo proposto e, desse modo, teriam que ser removidos ou outros itens deveriam ser incorporados ao modelo.

Inobstante a AFE não se tenha sido desconsiderada, algumas questões foram identificadas a partir das estatísticas descritivas referente a cada uma das variáveis componentes das dimensões investigadas no modelo de Popadiuk (2010).

No que diz respeito às práticas de conhecimento, foi detectada uma média relativamente baixa da variável PC_2 (uso de novas fontes de conhecimentos de parceiros), indicando, desse modo uma orientação exploiter. Em outro sentido, no que tange às práticas de inovação PI_8 (desenvolvimento de novos produtos 
e serviços), a média das respostas foi 5,28, considerada alta numa escala de 1 a 7 , o que indica uma orientação explorer. Isto denota que ambas as orientações (explorer e exploiter) estão presentes nos estabelecimentos investigados. Em outras variáveis analisadas, também se observou as duas orientações, o que faz concluir que não há apenas uma tendência das organizações.

Situações assim, denomina-se ambidestria, conforme Raisch et al. (2009), uma vez que organizações ambidestras tanto são capazes de aproveitar suas próprias competências, como conseguem explorar novas oportunidades.

Registre-se que uma das limitações desta pesquisa foi a quantidade da amostra estar no limite mínimo, ou seja, apenas 50 unidades, o que, talvez, tenha comprometido o resultado da análise fatorial exploratória. As dificuldades de aplicação do questionário junto aos empresários, apesar da boa vontade de muitos, são significativas. Muitas visitas e tentativas foram feitas para ampliar a quantidade amostral, contudo os resultados somente permitiram a amostra indicada. Nesse sentido, recomenda-se que outras investigações sejam realizadas, quais sejam: (a) o aumento da amostra pesquisada, possivelmente pela expansão geográfica da pesquisa, vez que o universo em Campina Grande é pequeno; (b) aplicação de pesquisas com abordagem qualitativa, as quais permitem um maior aprofundamento, tanto sobre inovação, quanto a respeito de explotação e exploração do conhecimento; (c) o emprego de investigações em outros segmentos econômicos, para avaliar melhor o modelo de Popadiuk (2010).

Por fim, consigne-se os resultados permitiram, em conformidade com os objetivos da pesquisa, alcançar o estabelecimento de uma análise sucinta das orientações exploiter e/ou explorer, no tocante às inovações dos bares e restaurantes de Campina Grande.

\section{REFERÊNCIAS}

ABERNATHY, W. J.; CLARK, K. B.. Innovation: mapping the winds of creative destruction. Research Policy, v.3, n.22, p.322, 1985.

BARNEY, J. B.; CLARK, D. N.. Resource-based theory: creating and sustaining competitive advantage. New York: Oxford University Press, 2007.

CORRÊA, H. L.; CORRÊA, C. A.. Administração da produção e operações. 3 ed. São Paulo: Atlas, 2012.

DAVENPORT, T. H.; PRUSAK, L.. Conhecimento empresarial: como as organizações gerenciam seu capital intelectual. 9 ed. Rio de Janeiro: Elsevier, 2004.

GUPTA, A. K.; SMITH, K. G.; SHALLEY, C. E.. The interplay between exploration and exploitation. Academy of Management Journal, v.49, n.4, p.693-706, 2006.

FANG, C.; LEE, J.; SCHILLING, M. A.. Balancing exploration and exploitation through structural design: the isolation of subgroups and organization learning. Organization Science, v.21, n.3, p.625-642, 2010.

FRANKLIN, M. A.. Estratégias organizacional, orientação para exploitation e exploration e tipos de inovação em institutos de pesquisa e desenvolvimento tecnológicos.
Tese (Doutorado em Administração de Empresas) Universidade Presbiteriana Mackenzie. São Paulo, 2010.

FREEMAN, C.. Introduction. In: DOSI, G.. Technical change and economic theory. London: Frances Printer, 1988.

HAIR JR., J. F.; BLACK, W. C.; BABIN, B. J.; ANDERSON, R. E.; TATHAM, R. L.; BLACK, W. C.. Análise multivariada de dados. 6 ed. Porto Alegre: Bookman: 2009.

HAUKNES, J.. Services in innovation - innovation in services. SI4S Project Synthesis. European Commission, through the Targeted Socio-Economic Research Programme (TSER): Oslo, 1998.

HITT, M.; IRELAND, R. D.; HOSKISSON, R. E.. Administração estratégica. São Paulo: Cencage Learning, 2008.

LEMOS, C.. A inovação na era do conhecimento. Parcerias Estratégicas, v. 8, p. 157-180, 2000.

LEVINTHAL, D. A.; MARCH, J. G.. The myopia of learning. Strategic Management Journal, v.14, p.95-112, 1993.

LIAO, S.. Knowledge management technologies and applications: literature review from 1995 to 2002. Expert Systems with Applications, v.25, p.155-164, 2003. 
MADEIRA, L. M. M.. Rumos da produção científica em gestão do conhecimento sob a ótica de seus relacionamentos com a gestão da inovação, da informação e da tecnologia. In.: SIMPÓSIO DE ENGENHARIA DE ENGENHARIA DE PRODUÇÃO, 14. Anais. Bauru: FEB/UNESP, 2012.

MALHOTRA, N. K.. Pesquisa de marketing: uma orientação aplicada. 6 ed. Porto Alegre: Bookman, 2010.

MARCH, J. G.. Exploration and exploitation in organizational learning. Organization Science, v.2, n.1, p.71-87, 1991.

MOM, T. J. M.: BOSCH, F. A. J. V. D.; VOLBERA, H. W.. Managing concurrently the processes of knowledge exploration and exploitation: the influence of knowledge exchange determinants and the introduction of an intranet. DRUID Summer Conference 2003 on creating, sharing and transferring knowledge. Anais. Copenhagen, 2003. p.12-14

NELSON, R. R.; WINTER, S. G.. An evolutionary theory of economic change. Cambridge: Harvard University Press, 1982.

NISHIMURA, C. S.. Orientação individual para exploração (exploration) e explotação (exploitation) e prontidão para tecnologia. Dissertação (Mestrado em Administração de Empresas) - Universidade Presbiteriana Mackenzie. São Paulo, 2012.

PASQUALI, L.. Análise fatorial: um manual teóricoprático. Brasília: EdUnB, 1999.

POPADIUK, S.. Exploration-exploitation de ativos do conhecimento: sobrevivência, paridade ou desempenho superior. In.: ENCONTRO DA ASSOCIAÇÃO NACIONAL DE PÓS-GRADUAÇÃO E PESQUISA EM ADMINISTRAÇÃO, 31. Anais. Rio de Janeiro: ANPAD, 2007.

POPADIUK, S; VIDAL, P. G.. Measuring knowledge exploitation and exploration: an empirical application in a technological development center in Brazil. In.: ENCONTRO DA ASSOCIAÇÃO NACIONAL DE PÓS-GRADUAÇÃO E
PESQUISA EM ADMINISTRAÇÃO, 33, 2009, São Paulo. Anais. Rio de Janeiro: ANPAD, 2009.

POPADIUK, S.. Escala de orientação exploration-exploitation do conhecimento em empresas brasileiras. In.: ENCONTRO DA ASSOCIAÇÃO NACIONAL DE PÓS-GRADUAÇÃO E PESQUISA EM ADMINISTRAÇÃO, 34, 2010, Rio de Janeiro. Anais. Rio de Janeiro: ANPAD, 2010.

RAISCH, S.; BIRKINSHAW, J.; PROBST, G.; TUSHMAN, M.. Organizational ambidexterity: balancing exploitation and exploration for sustained performance. Organization Science, v.20, n.4, p.685-695, 2009.

REICHERT, F. M.; CAMBOIM, G. F.; ZAWISLAK, P. A. Capacidades e trajetórias de inovação de empresas brasileiras. Revista de Administração da Mackenzie, v.16, n.5, p.161-194, 2015.

SEKARAN, U.. Research methods for business: a skill building approach. 4 ed. New Jersey: John Wiley \& Sons, 2003.

SILVEIRA-MARTINS, E.; ROSSETTO, C. R. Ambidestria organizacional - exploração e explotação: um estudo bibliométrico nas bases de dados internacionais. Revista Gestão Organizacional, v. 7, n. 2, p. 15-29, 2014.

SUNDBO, J.. Management of innovation in services. The Service Industries Journal, v.17, n.3, p.432-455, 1997.

SUNDBO, J.; GALLOUJ, F.. Innovation in services: SI4S Project Synthesis. European Commission, through the Targeted Socio-Economic Research Programme (TSER): Oslo, 1998.

ZACK, M. H.. Developing a knowledge. In.: CHOO, W.; BONTIS, N.. The strategic management of intellectual capital and organizational knowledge. New York: Oxford, 2002.

ZAWISLAK, P. A.; ALVES, A. C.; TELLO-GAMARRA, J.; BARBIEUX, D.; REICHERT, F. M.. Influences of the internal capabilities of firms on their innovation performance: a case study investigation in Brazil. International Journal of Management, v.30, n.1, p.329-348, 2013. 